\title{
Study on The Likelihood Ratio Used to Evaluate the Muscle Tension of Upper Limb Spasm in Hemiplegia Patients
}

\author{
Zhen $\mathrm{Wu}^{1 *}$, Hui Wang², Wanzhang Yang ${ }^{3}$, Guanglin $\mathrm{Li}^{2}$, Wuhua $\mathrm{Xu}^{1}$ \\ ${ }^{1}$ Guangzhou Red Cross Hospital, Guangzhou City, China \\ ${ }^{2}$ Shenzhen Institutes of Advanced Technology, Chinese Academy of Sciences, Shenzhen 518055, China \\ ${ }^{3}$ Shenzhen Hospital of Southern Medical University, Shenzhen City, China
}

*Corresponding author: Zhen Wu,wzhen8712@126.com

\begin{abstract}
Aim: To evaluate the clinical usability of mechanomyography (MMG) in the evaluation of upper-limb spasticity states of hemiplegia patients with likelihood ratio analysis. Methods: The MMG signals from the 30 hemiplegia patients' biceps and triceps were recorded with three-dimensional wireless accelerometer (Trigno Wireless System, Delsys Inc), when they extended or bent their elbow passively. At the same time, the physiotherapist assessed the MAS (Modified Ashworth Scale) of all the patients who participated in the study and would be divided them into four groups based on the MAS values (MAS0, MAS1, MAS1+ and MAS2). The MMG sensors were built with triaxial accelerometers named as $\mathrm{X}, \mathrm{Y}$ and $\mathrm{Z}$ that represent the muscle fibers lengthwise movement, the cross movement and the vertical the muscle moving direction, respectively. The root mean square (RMS) value of the MMG signal was calculated for analysis. Likelihood ratio analysis were used in the study. Results: All of the variables of the X, Y, Z axis of signals of MMG of BB and TB have related with muscle spasticity grading during passive elbow flexion in multinomial logistic regression $(\mathrm{P}=0.000, \mathrm{P}<0.001)$. The regression coefficient of $\mathrm{Y}$ axis signal of $\mathrm{MMG}$ of $\mathrm{BB}$ is the largest. The 5 variables of the $\mathrm{X}, \mathrm{Y}, \mathrm{Z}$ axis of signals of MMG of $\mathrm{BB}$ and $\mathrm{X}$, $\mathrm{Y}$ axis of TB have related with Muscle spasticity grading during passive elbow extension in multinomial logistic regression $(\mathrm{P}=0.000, \mathrm{P}<0.001)$. The regression coefficient of $\mathrm{Y}$ axis signal of $\mathrm{MMG}$ of $\mathrm{BB}$ is the largest in equation and the $\mathrm{Y}$ axis signal of MMG of TB is second large. Conclusions: The effect of agonist is more than the antagonist during the MAS assessment, especially the muscle fibers cross movement and the vertical movement by the MMG assessment.
\end{abstract}

Keywords: Mechanomyography; Muscle spasm; Modify Ashworth scale (MAS); Passive movement; Spasticity assessment

Publication date: July 2021; Online publication: July 31, 2021

\section{Introduction}

At present, there are many methods for assessing the spasticity of upper limbs of patients with hemiplegia. The contraction of muscle fibers is a three-dimensional dynamic process, and the muscle contraction vibration produces changes in the MMG signal of the myograph. In the previous study of the team, it was found that the myograph signal of elbow joint spasm in hemiplegic patients was correlated with the MAS score ${ }^{[6]}$. However, we did not further explore the reasons that may affect its correlation. The author guessed whether the myograph signals in the three different directions during muscle contraction all affect the MAS score. In response to this problem, this article intends to adopt the likelihood ratio based on the previous period. Statistical methods to explore the factors that affect the evaluation of upper limb muscle tension in patients with hemiplegia. 


\section{Information and method}

\subsection{General information}

From December 2019 to April 2021, 30 patients with hemiplegia after cerebrovascular disease were all inpatients from the Rehabilitation Department of Guangzhou Red Cross Hospital. The youngest was 27 years old and the oldest was 80 years old. Among them, 22 were males and 8 were females; at an average age. During the course of 5 years, 16 cases were left hemiplegia and 14 cases were right hemiplegia. All the patients understood the experimental process and signed an informed consent form.

\subsubsection{Standard constrains}

(1) Unconsciousness and severe cognitive impairment.

(2) Peripheral nerve damage in the upper limbs.

(3) Restriction of movement of the affected shoulder and elbow joints due to trauma, fracture, tissue damage, contracture, etc.

(4) Inability to maintain independent sitting and standing.

(5) Complete and sensory aphasia.

\subsubsection{Exclusion criteria's}

(1) Unconsciousness and severe cognitive impairment.

(2) Peripheral nerve damage in the upper limbs.

(3) Restriction of movement of the affected shoulder and elbow joints due to trauma, fracture, tissue damage, contracture, etc.

(4) Inability to maintain independent sitting and standing.

(5) Complete and sensory aphasia.

\subsection{Experimental data collection record}

\subsubsection{Experimental environment}

All patients were treated by the same physician in the same room, the room temperature was about 22-26 degrees. The patient was in a supine position, the shoulder joint of the affected limb was lifted at 90 degrees to the horizontal, and the elbow joint was in a natural anatomical position, completely relaxed.

\subsubsection{Experimental equipment}

German-made Trigno Wireless System delsys wireless acceleration sensor provided by Shenzhen Institute of Advanced Technology, Chinese Academy of Sciences. MMG sampling frequency was 148.1Hz, 16-bit resolution. Disposable double-sided electrode patch.

\subsubsection{Skin preparation}

The skin where the electrode was placed, was wiped back and forth with alcohol 5 times.

\subsubsection{Electrode placement}

The position of the wireless acceleration sensor was the measured muscle (the midpoint of the connection between the center of the biceps inner and outer head muscles and the midpoint of the connection between the center of the long head of triceps brachii to the centre of the short head of triceps brachii), allowing the $\mathrm{X}$ axis of the sensor to contract along the muscle fibers direction. Participants slowly performed passive elbow flexion and passive elbow extension on the test side, with an angular velocity of about 60 degrees/s, assessment of muscle tension, and recording of myograph at the same time. The doctor performed a modified Ashworth assessment with bare hands and used delsys to record the muscle signals of the biceps and triceps. Fixed the elbow joint with one hand and held the wrist joint with the other hand. The passive range of elbow joint motion was from 0 to 120 degrees. First, 3 passive elbow flexion was done, then 3 passive elbow extension was done. Each passive activity should be completed at a uniform speed as much as possible, the time was 3 seconds, and the interval between the two was 8 seconds. 


\subsubsection{Record indicators and data processing}

Recorded the biceps and triceps muscle signals, analyzed them with MATLAB software, took 3 complete original signals, and performed root-mean-square (RMS) conversion with a period of $1 \mathrm{~s}$. In passive elbow extension and passive elbow flexion, the correlation between the RMS value of the muscle vibration of the biceps and triceps contraction and MAS was analyzed in the form of X, Y, and Z axes, and the X axis was the muscle fiber in the longitudinal movement direction, the $Y$ axis was the transverse vibration direction of the muscle fibers, and the $\mathrm{Z}$ axis was the direction perpendicular to the muscle surface.

\subsection{Statistical analysis}

The RMS measurement value was expressed as ( $\overline{\mathrm{x}} \pm \mathrm{s})$. Using SPSS17.0 statistical software, the RMS values of the $\mathrm{X}, \mathrm{Y}$, and $\mathrm{Z}$ axes of passive elbow flexion and passive elbow extension MMG were tested with MAS respectively, and the significance level was set to $\alpha=0.05$.

\subsection{Experimental results}

First, calculated the RMS value of the MMG of each level of the MAS group when the biceps and triceps were passive elbow flexion and passive elbow extension, and then calculated the $\mathrm{X}, \mathrm{Y}$, and $\mathrm{Z}$ three axes of the passive elbow flexion and passive elbow extension MMG. The RMS value was tested with the MAS for likelihood ratio. The results of passive elbow flexion are shown in Table C1. When passively extending the elbow, the results are shown in Table D1. Table C2. and Table D2. are the estimation results of multiple logit model parameters during passive elbow flexion and elbow extension.

Likelihood ratio (LR) is an indicator that reflects authenticity, and it is a composite indicator that reflects both sensitivity and specificity. Likelihood ratio combines the characteristics of sensitivity and specificity, and is not affected by the prevalence. It is a relatively stable comprehensive index.

Table C1. The likelihood ratio test of the six independent variables on the $\mathrm{X}, \mathrm{Y}$, and $\mathrm{Z}$ axes of passive elbow flexion sensors 1 and 2

\begin{tabular}{|c|c|c|c|c|}
\hline Effect & $\begin{array}{l}-2 \text { Log Likelihood of } \\
\text { Reduced Model }\end{array}$ & $\begin{array}{l}\text { Chi- } \\
\text { Square }\end{array}$ & df & Sig \\
\hline $\begin{array}{l}\text { Passive elbow flexion sensor } 1 \mathrm{X} \\
\text { axis signal }\end{array}$ & 134.458 & 23.306 & 3 & .000 \\
\hline $\begin{array}{l}\text { Passive elbow extension sensor } 2 \mathrm{X} \\
\text { axis signal }\end{array}$ & 122.431 & 11.279 & 3 & .010 \\
\hline $\begin{array}{c}\text { Passive elbow bending sensor } 1 \mathrm{Y} \\
\text { axis signal }\end{array}$ & 165.862 & 54.711 & 3 & .000 \\
\hline $\begin{array}{l}\text { Passive elbow extension sensor } 2 \mathrm{Y} \\
\text { axis signal }\end{array}$ & 145.747 & 34.596 & 3 & .000 \\
\hline $\begin{array}{l}\text { Passive elbow flexion sensor } 1 \mathrm{Z} \\
\text { axis signal }\end{array}$ & 166.320 & 55.169 & 3 & .000 \\
\hline $\begin{array}{c}\text { Passive elbow extension sensor } 2 \mathrm{Z} \\
\text { axis signal }\end{array}$ & 133.728 & 22.577 & 3 & .000 \\
\hline
\end{tabular}


Table C2. Multinomial logit model parameter estimation results of passive elbow flexion

\begin{tabular}{|c|c|c|c|c|c|c|}
\hline \multirow{2}{*}{ Effect } & \multicolumn{2}{|c|}{$\mathbf{M A S}=0$} & \multicolumn{2}{|c|}{ MAS $=1$} & \multicolumn{2}{|c|}{ MAS $=1+$} \\
\hline & B & Sig & B & Sig & B & Sig \\
\hline $\begin{array}{l}\text { Passive elbow flexion sensor } 1 \mathrm{X} \text { axis } \\
\text { signal }\end{array}$ & 11.453 & .593 & -2.622 & .901 & 5.642 & .793 \\
\hline $\begin{array}{l}\text { Passive elbow extension sensor } 2 \mathrm{X} \text { axis } \\
\text { signal }\end{array}$ & -32.897 & .091 & -32.603 & .091 & -12.729 & .534 \\
\hline $\begin{array}{l}\text { Passive elbow bending sensor } 1 \mathrm{Y} \text { axis } \\
\text { signal }\end{array}$ & -206.083 & .046 & -219.327 & .034 & -165.761 & .107 \\
\hline $\begin{array}{c}\text { Passive elbow extension sensor } 2 \mathrm{Y} \text { axis } \\
\text { signal }\end{array}$ & -174.818 & .046 & -177.198 & .043 & -142.436 & .104 \\
\hline $\begin{array}{l}\text { Passive elbow flexion sensor } 1 \mathrm{Z} \text { axis } \\
\text { signal }\end{array}$ & -52.928 & .071 & -65.469 & .026 & -33.175 & .263 \\
\hline $\begin{array}{l}\text { Passive elbow extension sensor } 2 \mathrm{Z} \text { axis } \\
\text { signal }\end{array}$ & 107.777 & .068 & 102.736 & .081 & 115.817 & .050 \\
\hline
\end{tabular}

Table D1. The likelihood ratio test of the six independent variables of the $\mathrm{X}, \mathrm{Y}$, and $\mathrm{Z}$ axes of passive elbow extension sensors 1 and 2

\begin{tabular}{ccccc}
\hline Effect & $\begin{array}{c}-\mathbf{2} \text { Log Likelihood } \\
\text { of Reduced Model }\end{array}$ & Chi-Square & df & Sig \\
\hline Passive elbow flexion sensor 1 X axis signal & 162.797 & 16.179 & 3 & .001 \\
Passive elbow extension sensor 2 X axis signal & 156.804 & 10.185 & 3 & .017 \\
Passive elbow bending sensor 1Y axis signal & 155.478 & 8.859 & 3 & .031 \\
Passive elbow extension sensor 2 Y axis signal & 165.334 & 18.715 & 3 & .000 \\
Passive elbow flexion sensor 1 Z axis signal & 166.696 & 20.077 & 3 & .000 \\
Passive elbow extension sensor 2 Z axis signal & 150.585 & 3.966 & 3 & .265 \\
\hline
\end{tabular}


Table D2. Parameter estimation results of passive elbow extension multinomial logit model

\begin{tabular}{|c|c|c|c|c|c|c|}
\hline \multirow{2}{*}{ Effect } & \multicolumn{2}{|c|}{$\mathbf{M A S}=\mathbf{0}$} & \multicolumn{2}{|c|}{ MAS=1 } & \multicolumn{2}{|c|}{ MAS $=1+$} \\
\hline & B & Sig & B & Sig & B & Sig \\
\hline $\begin{array}{l}\text { Passive elbow flexion sensor } 1 \mathrm{X} \\
\text { axis signal }\end{array}$ & 10.708 & -12.211 & -24.926 & -20.160 & -15.803 & 6.406 \\
\hline $\begin{array}{l}\text { Passive elbow extension sensor } 2 \mathrm{X} \\
\text { axis signal }\end{array}$ & .252 & .213 & .025 & .045 & .022 & .375 \\
\hline $\begin{array}{l}\text { Passive elbow bending sensor } 1 \mathrm{Y} \\
\text { axis signal }\end{array}$ & 20.585 & -4.530 & -19.735 & -16.971 & -14.023 & .669 \\
\hline $\begin{array}{l}\text { Passive elbow extension sensor } 2 \mathrm{Y} \\
\text { axis signal }\end{array}$ & .023 & .580 & .065 & .087 & .034 & .923 \\
\hline $\begin{array}{l}\text { Passive elbow flexion sensor } 1 \mathrm{Z} \\
\text { axis signal }\end{array}$ & 16.350 & 4.250 & -16.101 & -6.338 & -1.742 & 1.711 \\
\hline $\begin{array}{l}\text { Passive elbow extension sensor } 2 \mathrm{Z} \\
\text { axis signal }\end{array}$ & .069 & .616 & .136 & .547 & .814 & .806 \\
\hline
\end{tabular}

Table C1. shows the likelihood ratio test results show that these six variables are related to the classification of passive elbow flexion spasticity $(\mathrm{p}<0.05)$. In the logit parameter equation of Table C2., it is shown that in level 0 , the predictive variables with significant significance are s1y_eb and s2y_eb. In level 1, significant predictors include s1y_eb, s2y_eb, and s1z_eb. In level 1+, the significant predictor variable is $\mathrm{s} 2 \mathrm{z} \_\mathrm{eb}$. It can be seen from the size of the coefficient that the regression coefficient of the $\mathrm{y}$-axis is larger, which indicates that the lateral movement has a greater influence on the classification of spasticity.

Table D1. shows the likelihood ratio test results, it shows that these five variables (passive elbow extension sensor $1 \mathrm{X}$-axis, $\mathrm{Y}$-axis, $\mathrm{Z}$-axis signal, passive elbow extension sensor $2 \mathrm{X}$-axis, $\mathrm{Y}$-axis signal) and passive elbow extension spasticity state classification that there is a relationship $(p<0.05)$. Furthermore, it suggests that the independent variable passive elbow extension sensor $2 \mathrm{Z}$-axis signal may have nothing to do with the degree of spasticity during passive elbow extension $(p=0.265)$, but it may be caused by a non-linear relationship between the two, but it doesn't matter.

Table D2. from the Logit parameter equation, it can be seen that in level 0 , the significant predictor variable is passive elbow extension sensor 1. Axis signal, passive elbow extension sensor $2 \mathrm{Y}$-axis signal, passive elbow extension sensor $1 \mathrm{Z}$-axis signal. In level 1 , significant predictors include passive elbow extension sensor $1 \mathrm{X}$-axis signal, passive elbow extension sensor $1 \mathrm{Z}$-axis signal. In level $1+$, there is no significant predictor variable.

\subsubsection{Note}

(1) s1 means, the sensor is attached to the skin surface of the biceps brachii.

(2) s2 means, the sensor is attached to the skin surface of the triceps brachii.

(3) $\mathrm{x}$ behind s1 and s2 represents the direction along the muscle contraction. 
(4) y behind s1 and s2 represents the direction perpendicular to the muscle contraction direction.

(5) $\mathrm{z}$ behind $\mathrm{s} 1$ and $\mathrm{s} 2$ represents the direction perpendicular to the $\mathrm{x}$-axis.

(6) eb stands for passive elbow flexion.

(7) ee stands for passive elbow extension.

\section{Discussion}

The MMG signal was mainly manifested in the mechanics as the mechanical vibration generated by the muscle fiber after activating the motion unit and contracting, which was generally the a low-frequency lateral vibration of skeletal muscle fiber movement ${ }^{[7-8]}$. The greater the muscle contraction force, the greater the amplitude of MMG RMS ${ }^{[8]}$. The MAS score (modified Ashworth score) was a subjective assessment method that was currently used in clinical practice. It was used by the physician to passively move the patient's joints to stretch the muscles of the spasm, and feel the range and intensity of the joint movement resistance to determine the degree of spasticity.

The team's previous research found ${ }^{[6]}$ that during the MAS evaluation process, the three-dimensional space of the muscle group, namely the part of the muscle fiber vibration in the $\mathrm{X}, \mathrm{Y}$, and $\mathrm{Z}$ axis, were correlated with the MAS score. The score of MAS in passive elbow flexion was affected by both the active biceps brachii muscles and the triceps antagonist muscles. However, in the process of completing the passive elbow flexion, the active biceps brachii muscles play a more important role. The elements of this were not statistically significant to the correlation between the movement displacement of the muscle fibers of the triceps brachii muscle and its lateral vibration, but it was related to the vertical vibration of the muscle, and the relationship was the greatest. In passive elbow extension, only the $\mathrm{Y}$-axis and $\mathrm{Z}$-axis of the active muscles are correlated with the MAS score.

In order to better explain that there was indeed a correlation between MMG signal and MAS score, and that there should be one or several factors in the directed axis of muscle fiber movement that have the greatest impact on MAS score. Two-class logic regression and multinomial logistic regression was commonly used in medical research, especially in epidemiological research. At present, the MAS research on spasticity in China and abroad has not found the use of multinomial variable logistic regression analysis, but the author believes that the MAS score starts from level 0, level 1, level 1+, level 2, and level 3. This is a continuous grade variable; completely ordinal multinomial logistic regression can be used to establish a logistic regression equation to analyze the largest contributor to the MAS score.

Logistic regression for ordered classification uses a multinomial logistic model. If the dependent variable $\mathrm{Y}$ has $\mathrm{M}$ values (that is, Y has M categories), one of the categories is used as the reference category (baseline category), and the other categories are the same, then in comparison, M-1 non-redundant logistic transformation models can be generated. For example, the dependent variable is $\mathrm{M}$ category, $\mathrm{N}$ independent variables $\mathrm{X}$, the logistic model uses $\mathrm{Y}=\mathrm{M}$ as the $\mathrm{Y}$ reference category, then for the $\mathrm{Y}=\mathrm{i}$ category, the logistic transformation model is:

$$
G=\log \frac{P(Y=i)}{P(Y=M)}=\alpha+\beta_{i 1} X_{1}+\beta_{i 2} X_{2}+\ldots+\beta_{i N} X_{N}
$$

For the reference category $\mathrm{Y}=\mathrm{M}, \mathrm{G}=0$, all the coefficients in the model are 0 . In this study, the spasticity of the collected subjects has four levels: $0,1,1+$, and 2 For multiple logistic model reference classes, it is generally necessary to consider whether its meaning is clear, and its sample size cannot be too different from others. The higher MAS level 2 is clinically easier to diagnose, with less contradictions and smaller standard deviation. It is also the middle level in the entire MAS score. Therefore, the category with spasticity level 2 is used as the reference category, namely MAS $=2$ is the reference group. 
It can be seen that in the process of passive elbow flexion, the likelihood ratio test results in Table C1. and passive elbow extension Table D1. indicate that there is a relationship between the MMG signal and the MAS classification of elbow spasticity $(\mathrm{p}<0.05)$. Although shown in Table D1. that the independent variable passive elbow extension sensor $2 \mathrm{Z}$-axis signal may have nothing to do with the degree of spasticity during passive elbow extension ( $\mathrm{p}=0.265$ ), it may be caused by the non-linear relationship between the two, which is not unrelated. In addition, from the logit parameter equation in Table C2. it is shown that in level 0 , the significant predictor variables are the $\mathrm{Y}$-axis signals of passive elbow extension sensors 1 and 2 (the amplitude of the lateral vibration of the biceps and triceps is significant "significance") In level 1 , the predictive variables with significant significance include passive elbow extension sensor 1 and $2 \mathrm{Y}$-axis signal, passive elbow extension sensor $1 \mathrm{Z}$-axis signal, that is, one more factor of the vertical vibration direction signal of the active muscle than level 0 This is basically close to the result of spearman correlation analysis of MMG signal and MAS score.

Similar results were found in passive elbow extension. At the same time, it was observed that regardless of elbow extension or elbow flexion, the regression coefficient $\mathrm{B}$ in the equation is the sensor $1 \mathrm{Y}$ axis signal> sensor $2 \mathrm{Y}$ axis signal> sensor $2 \mathrm{Z}$ axis signal, which shows that the MAS score follows the relationship between the lateral vibration of muscles is relatively large, that is, the lateral vibration of muscles has a greater impact on the score of MAS. The RMS values of the MMG of the biceps brachii and triceps brachii in Table 1. of the MMG amplitudes of the spearman correlation analysis found in the previous period are higher than those of the $\mathrm{X}$-axis and Z-axis, and gradually increases with the level. This is consistent with the electromyographic signal characteristics of the active and antagonist muscles in passive elbow flexion done by Mingyuan Guo of Southern Medical University. It further shows that there is a correlation between the MMG signal and the MAS score, and the lateral vibration of the muscle is an important factor affecting the MAS score and the severity of elbow joint spasm. This may provide us with a new path for the subsequent clinical treatment of spasticity.

\section{Acknowledgments}

Thanks to Shenzhen Institute of Advanced Technology, Chinese Academy of Sciences for providing Delsys collectors.

\section{Funding}

This work was partially funded by the National Natural Science Foundation of China (\#61135004, \#51275101).

\section{Disclosure statement}

The author declares no conflict of interest.

\section{References}

[1] Kawczyński A, Nie H, Jaskolska A, et al., 2007, Mechanomyography and Electromyography During and After Fatiguing Shoulder Eccentric Contractions in Males and Females. Scandinavian Journal of Medicine \& Science in Sports, 17(2): 172-179.

[2] Tarata MT, 2003, Mechanomyography Versus Electromyography, in Monitoring the Muscular Fatigue. Biomed Eng Online, 2(3).

[3] You M. 2011, Research on Prosthetic Control System Based on Myograph (MMG) and Electromyography (EMG) Signals. Central South University, 2-5. 
[4] Alves-Kotzev N, 2010, Mechanomyography as an Access Pathway for Binary and Multifunction Control. University of Toronto.

[5] Wu Z, Wang H, Wang J, et al., 2014, Myograph Assessment of Elbow Joint Spasticity in Patients with Hemiplegia After Stroke. Journal of Integrated Traditional Chinese and Western Medicine Cardio-Cerebrovascular Disease, (12):1456-1458.

[6] Veltink P, Slycke H, et al., Three-dimensional inertial sensing of foot movements for automatic tuning of a two-channel implantable drop-foot stimulator. Medical Engineering \& Physics .2003:25,21-28.

[7] Antonelli MG, Zobel PB, Giacomin J, 2009, Use of MMG Signals for the Control of Powered Orthotic Devices: Development of a Rectus Femoris Measurement Protocol. Assist Technology 2009 Spring, 21(1):1-12.

[8] Shinohara M, Sogaard K, 2006, Mechanomyography for Studying Force Fluctuations and Muscle Fatigue. Exercise and Sport Sciences Reviews, 34(2):59-64. 